\title{
Review: psychodynamic therapy and cognitive behavioural therapy are effective in the treatment of personality disorders
}

Leichsenring F, Leibing E. The effectiveness of psychodynamic therapy and cognitive behavior therapy in the treatment of personality disorders: a meta-analysis. Am J Psychiatry 2003; 160:1223-32.

\section{$Q$ Are psychodynamic therapy and cognitive behaviour therapy effective treatments for people with personality disorders?}

METHODS

Design: Systematic review with meta-analysis.

Data sources: MEDLINE, PsycINFO, and Current Contents

然

(11) Study selection and analysis: Eligible studies diagnosed personality disorders using standardised methods, assessed outcomes using reliable validated instruments, and presented data in a way that permitted calculation of within-group effect sizes or assessment of recovery rates. Details of data extraction were not reported. Longest post-treatment follow up was used to assess long term outcomes. Within-group effect sizes were calculated using Cohen's d. In addition to overall effect sizes, individual effect sizes were calculated for measures that were more specific to personality disorders. Effect sizes were calculated separately for self and observer rated measures. Rates of improvement were assessed where possible. Correlation between outcome and length of therapy, participant sex, hospitalisation status, use of therapy manuals, reported experience of therapists, and study design were calculated.

Outcomes: Effectiveness of psychodynamic therapy and cognitive behaviour therapy.

\section{MAIN RESULTS}

14 studies of psychodynamic therapy (417 participants; includes 6 RCTs; mean follow up 78 weeks) and 11 studies of cognitive behaviour therapy (231 participants; includes 5 RCTs; mean follow up 13 weeks) met inclusion criteria. For psychodynamic therapy the mean overall effect size was significantly greater than zero (1.46; $\mathrm{SD}=0.73 ; \mathrm{p}=0.0001$ ). In 2 RCTs comparing psychodynamic therapy with control, psychodynamic therapy was significantly more effective. For cognitive behaviour therapy the mean overall effect size was significantly greater than zero $(1.0 ; \mathrm{SD}=0.48 ; \mathrm{p}=0.0001)$. Cognitive behaviour therapy was significantly more effective than control in 3 out of 5 RCTs.

\section{CONCLUSIONS}

Preliminary evidence suggests that both psychodynamic therapy and cognitive behavioural therapy are effective in the treatment of personality disorders.
For correspondence: Dr Leichsenring, Department of Psychosomatics and Psychotherapy, University of Grottingen, von-Siebold-str, Grottigen, Germany; fleichs@gwdg.de

Source of funding: not specified.

\section{NOTES}

Although all studies used validated diagnostic criteria, seven different diagnostic instruments were used. Number of sessions and duration of follow up varied between studies. Authors note that the small number of studies reduces the power of the meta-analysis and the potential for generalisation of findings.

\section{Commentary}

$\mathrm{T}$ en to 15 percent of the adult population has a personality disorder (PD), which carries with it significant Axis I comorbidity, distress, psychosocial role impairment, and mental health service use. This underlines the importance of the authors' examination of two frequently used psychotherapies for personality disorders: dynamic and cognitive behavioural (CBT) psychotherapy. They largely address questions and follow the quantitative methods and analyses presented in earlier metaanalyses conducted on a more heterogeneous sample of psychotherapies. ${ }^{12}$ Like those reviews, they report positive evidence of both effectiveness and efficacy. The major contribution of Leichsenring and Leibing is the specification that both dynamic and CBT therapies have sizable effects. This is true for both cohort and the few RCT studies as previously found. 'Although somewhat larger effects were found in observer rated compared to self report instruments, this may be due to differences in measurement constructs, state dependent characteristics, as well as perspective.

Generally, dynamic psychotherapy produced larger effect sizes than CBT. However, this was confounded by differences regarding specific PD diagnoses, measures, treatment durations, and follow up. Unfortunately, several head-to-head randomised comparisons were limited by short treatment durations. Future RCTs of sufficient duration are needed to determine the comparative efficacy of the two therapies, hopefully in comparison to also widely used supportive therapies.

For those who missed the message from the earlier reviews, it is clear that these two common psychotherapies for PDs work. Left for future study is the relationship of specific PD type or other patient characteristics to optimal treatment duration, although existing evidence suggests that durations greater than one year will generally be needed. Additional studies of cost effectiveness are required, as is greater emphasis on assessing underlying personality functioning and what constitutes recovery, and determining whether certain patients respond differentially to these therapies. While therapies for PDs require further refinement and testing, the evidence in this report cautions against a rush to embrace a single treatment (often CBT) and duration (usually short) by programme administrators and grant review committees.

Prof J Christopher Perry, MPH, MD

Department of Psychiatry, McGill University at SMBD Jewish General Hospital, Montreal, Canada

1 Perry JC, Banon L, lanni F. The effectiveness of psychotherapy for personality disorders. Am J Psychiatry 1999;156:1312-21.

2 Perry JC, Bond M. Empirical studies of psychotherapy for personality disorders. In: Gunderson JG, Gabbard GO, eds. Psychotherapy of Personality Disorders. (Review of Psychiatry Series, Vol 19, No 3, Oldham JM, Riba MB, Series eds). Washington, DC: American Psychiatric Press, 2000:1-31. 